\title{
Genetic and biochemical biomarkers related to oxidative stress in patients with schizophrenia
}

L. Forner $^{1^{*}}$ T T Andreguetto ${ }^{1^{*}}$, M. opes $^{1}$ P. Buosi ${ }^{1}$ F A. Borghi ${ }^{2}$ I.S. Facincani ${ }^{1}$, R. Fernandes-Ferreira ${ }^{7,8}$, C.I.F. Oliveira-Brancati ${ }^{1}$, E.A. de Almeida ${ }^{3}$, A.A. Felício ${ }^{4}$, D.P. Martins ${ }^{1}$, G.M.A. Filho ${ }^{2,5}$ and D.R.S. Souza ${ }^{1,6}$

* These authors have equally contributed to this study

${ }^{1}$ Núcleo de Pesquisa em Bioquímica e Biologia Molecular, Faculdade de Medicina de São José do Rio Preto, São José do Rio Preto, SP, Brasil

${ }^{2}$ Hospital de Base, Faculdade de Medicina de São José do Rio Preto, São José do Rio Preto, SP, Brasil

${ }^{3}$ Departamento de Ciências Naturais, Universidade Regional de Blumenau, Blumenau, SC, Brasil

${ }^{4}$ Universidade Estadual Paulista, São José do Rio Preto, SP, Brasil

${ }^{5}$ Departamento de Psiquiatria e Psicologia Médica, Faculdade de Medicina de São José do Rio Preto, São José do Rio Preto, SP, Brasil

${ }^{6}$ Departamento de Biologia Molecular, Faculdade de Medicina de São José do Rio Preto, São José do Rio Preto, SP, Brasil

${ }^{7}$ Universidade Estadual de Campinas, Campinas, SP, Brasil

${ }^{8}$ Universidade Paulista, São José do Rio Preto, SP, Brasil

Corresponding author: D.R.S. Souza

E-mail: doroteia@ famerp.br

Genet. Mol. Res. 18 (2): gmr18259

Received February 10, 2019

Accepted May 17, 2019

Published May 24, 2019

DOI http://dx.doi.org/10.4238/gmr18259

ABSTRACT. We evaluated the association of glutathione Stransferase polymorphisms (GSTT1 and GSTM1) and oxidative stress biomarkers in patients with schizophrenia. A total of 162 subjects were studied: 53 had schizophrenia (Total Study Group-TSG), and 109 without the disorder (Total Control Group-TCG). To analyze oxidative stress, TSG was distributed into treatment-responsive schizophrenia $(\mathrm{N}=26)$ and treatment-resistant $(\mathrm{N}=27)$ versus 
control group $(\mathrm{N}=36)$. Peripheral blood collection for analysis of polymorphisms, malondialdehyde (MDA) and trolox equivalent antioxidant capacity (TEAC); a questionnaire or a medical record for clinical profile and lifestyle was also applied. Frequency of genotypes did not differ significantly between the groups. The patients had a significantly reduced frequency of the combination GSTT1-null/GSTM1-present (13 versus 30\%) and significantly higher plasma MDA levels, but similar TEAC values. Smoking, diabetes mellitus (DM), systemic arterial hypertension (SAH) and family history (FH) significantly prevailed in patients (TSG) compared to controls (TCG). High sensitivity and specificity values for MDA (area under the curve >0.90) were observed. Reduced frequency of the combination GSTT1-null/GSTM1-present in patients suggests exposure to oxidative stress, represented by increased MDA and mainly aggravated by smoking, SAH, DM and FH. High sensitivity and specificity identifies the potential of MDA as a marker of oxidative stress in schizophrenia.

Key words: Schizophrenia; Biochemical markers; Oxidative stress; Genetic polymorphisms

\section{INTRODUCTION}

Schizophrenia, a severe mental disorder, is characterized by neurological, behavioral and emotional changes. The disease affects $1 \%$ of the world population, mainly individuals under 25 years of age (Kim et al., 2015; Pinheiro et al., 2017; Shojaeimotlagh et al., 2019). Genetic, environmental and metabolic factors have been associated with this disorder (Boskovic et al., 2011; Emiliani et al., 2014; Mak et al., 2019; Shojaeimotlagh et al., 2019).

Genetic alterations of glutathione S-transferases (GSTs), a family of isoenzymes with antioxidant effects that regulates the transformation of toxic compounds, such as oxygen reactive species into hydrophilic metabolites, increases susceptibility to schizophrenia associated with oxidative stress (Kim et al., 2015; Hu et al., 2019). In this case, the nullity of the GSTM1 genotype can be pointed out as a risk factor for the disorder even in the presence of the GSTT1 genotype (Koga et al., 2014; Nakamura et al., 2015). Oxidative stress, an imbalance between reactive oxygen species and antioxidant agents, can be measured also by biomarkers such as malondialdehyde (MDA), a byproduct of lipid peroxidation that impairs the functioning of neuronal cells (Emiliani et al., 2014; Liencres et al., 2014; Pawar and Abhang, 2017; Zheng et al., 2019). Additionally, the competence of antioxidant components can be evaluated by the trolox equivalent antioxidant capacity (TEAC) (Gilca et al., 2014; Filho et al., 2018).

We hypothesized that a combination of genetic and biochemical factors can help to identify new biomarkers for schizophrenia, since some studies have shown association with oxidative stress (Liencres et al., 2014; Reyezuddin et al., 2014). Thus, 
we aimed at analyzing genetic polymorphisms of GSTs (GSTM1 and GSTT1) and plasma levels of MDA and TEAC in patients with treatment-responsive and treatment resistant schizophrenia, in addition to information about their clinical profile and lifestyle.

\section{MATERIAL AND METHODS}

A total of 162 participants were divided into Total Study Group (TSG: N = 53 patients with schizophrenia) distributed in treatment-responsive (SG-1: $\mathrm{N}=26$ ) and treatment-resistant (SG-2: N = 27), and Control Group (Total Control Group - TCG: N $=109$ subjects without the disorder; a subgroup was sampled for analysis of oxidative stress - Control Group Oxidative Stress - CG-OS: N = 36). Resistance to drug treatment was defined according to the criteria established by Kane et al. (1988), that is, with persistent moderate to severe positive symptoms, even during the treatment period with antipsychotic- recommended doses for pre-established periods (four to six weeks) (Kane et al., 1988). Exclusion criteria included pregnancy, the use of nutritional supplements, hydroxycarbamide, opioids and medications such as antibiotics, antiinflammatories, acetylsalicylic acid and vitamins, blood transfusion within 120 days prior to starting the study, diagnosis of acute complications in the previous 12 months, presence of other psychotic disorders and lack of diagnostic criteria for schizophrenia.

All participants were submitted to blood sample collection and filled out a questionnaire with information about demographic profile, lifestyle (smoking, alcohol consumption and use of illicit drugs), comorbidities (diabetes mellitus - DM and systemic arterial hypertension - SAH) and familial history, and also medical records were consulted.

Polymorphisms of the GSTM1 and GSTT1 were analyzed by PCR using CYPcontrol (Salazar et al., 1998; Rossini et al., 2002). Plasma levels of MDA and TEAC were analyzed by high performance liquid chromatography and spectrophotometry (Liencres et al., 2014; Gilca et al., 2014), respectively, in all patients $(\mathrm{N}=53)$, and in the subgroup of control subjects $(\mathrm{N}=36)$.

Fisher's exact test or a Chi-square test and logistic regression were applied for categorical variables, and the Kruskal Wallis and Mann-Whitney tests for biomarkers of oxidative stress and age. The receiver operating characteristic curve (ROC) was used to calculate the cutoff point for the combination of sensitivity and specificity considering an area under the curve (AUC) of 0.70 for MDA and TEAC. Alpha-error was set to 5\%.

This study was approved by the Research Ethics Committee of the Medical School of São José do Rio Preto-FAMERP (CAAE: 20529913.7.0000.5415). The participants signed informed consent forms.

\section{RESULTS}

Patients and controls had a prevalence of males, older age, as well as smoking, SAH, DM and familial history highlighted in the patients (TSG), also shown by logistic regression ( $\mathrm{P}<0.05$; data not shown), comparing to controls (TCG; $\mathrm{P}<0.05$; Table 1). 
The frequency of the GSTM1 and GSTT1 genotypes was similar in the two groups (P > $0.05)$, but the patients showed a lower frequency of the combination of genotypes GSTT1-null/GSTM1-present (13\% versus TCG $=30 \% ; \mathrm{P}=0.0301$; Table 1).

Plasma MDA levels were increased in patients (median: SG-1 $=154.2 \mathrm{ng} / \mathrm{mL}$; SG-2 = $151.4 \mathrm{ng} / \mathrm{mL}$ ) compared to controls (median: $124.8 \mathrm{ng} / \mathrm{mL} ; \mathrm{P}<0.0001$; Figure 1A), but similar for TEAC $(\mathrm{P}=0.7513$, Figure 1B). According to genotype combination MDA levels were increased in patients in the presence of both genotypes $(G S T T 1 / G S T M 1:$ median $=151.8 \mathrm{ng} / \mathrm{mL})$ and $G S T T 1$-present $/ G S T M 1$-null $($ median $=$ $154.1 \mathrm{ng} / \mathrm{mL}$ ), compared with controls (median = $123.1 \mathrm{ng} / \mathrm{mL} ; 123.9 \mathrm{ng} / \mathrm{mL}$, respectively; $\mathrm{P}<0.0001$; Figure 1C). TEAC values were similar between groups $(\mathrm{P}>$ 0.05; Figure 1D).
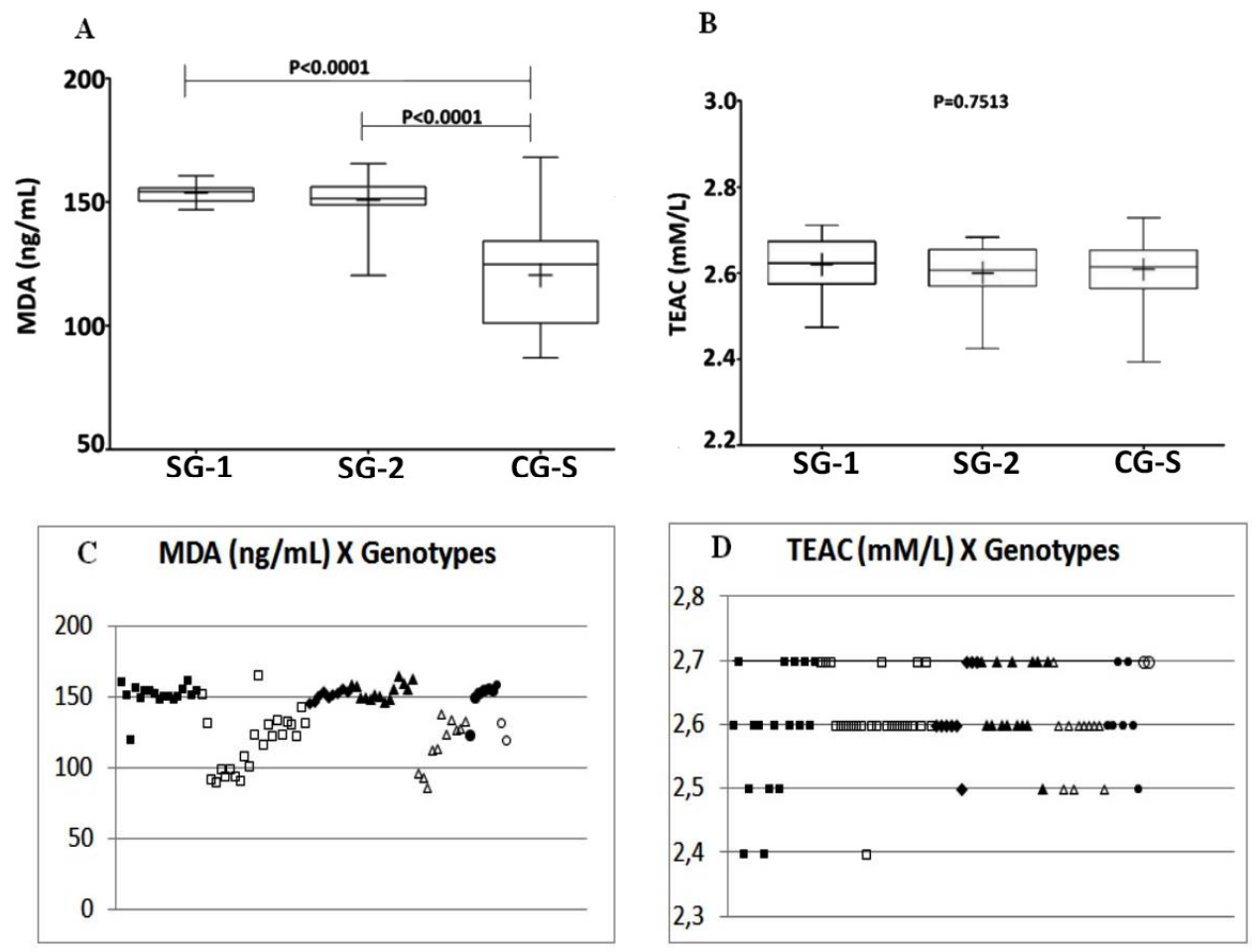

Figure 1. Plasma levels of (A) malondialdehyde (MDA) and (B) trolox equivalent antioxidant capacity (TEAC) in patients with schizophrenia treatment-responsive (SG-1) and resistant (SG-2) compared to controls with oxidative stress analyses (CG-OS). Distribution of MDA and TEAC levels for the total study group (TSG) and control group oxidative stress (CG-OS) according to combination of genotypes is showed in the Figures C and D, respectively. Legend: + = mean value; $\mathbf{m}:$ TSG = GSTT1-present/GSTM1-present; $\square:$ CG-OS = GSTT1present/GSTM1-present; $\mathbf{\Delta}:$ TSG = GSTT1-present/GSTM1-null; $\Delta:$ CG-OS = GSTT1-present/GSTM1-null; $:$ TSG = GSTT1-null/GSTM1null; $\circ:$ CG-OS = GSTT1-null/GSTM1present; • : TSG = GSTT1-null/GSTM1present. C) $\mathrm{P}$-significant value: $\mathbf{\boldsymbol { x }} \square=\mathrm{P}<0.0001 ; \boldsymbol{\Delta} \mathrm{X} \Delta=\mathrm{P}<0.0001$. 
Table 1. Demographic profile, lifestyle, comorbidities and genotypic frequencies of glutathione $\mathrm{S}$ transferases polymorphisms GSTT1 and GSTM1 in the total of patients with schizophrenia (TSG), and distributed in groups considering treatment-responsive (SG-1) and treatment-resistant (SG-2), besides the total control group (TCG) represented also by a subgroup for the stress oxidative analysis (CG-OS).

\begin{tabular}{|c|c|c|c|c|c|c|c|c|c|}
\hline & $\begin{array}{l}\text { TSG (a) } \\
\mathrm{N}=53\end{array}$ & $\begin{array}{l}\text { SG-1 (b) } \\
\mathrm{N}=26\end{array}$ & $\begin{array}{l}\text { SG-2 (c) } \\
\mathrm{N}=27\end{array}$ & $\begin{array}{l}\text { TCG (d) } \\
\mathrm{N}=109\end{array}$ & $\begin{array}{l}\text { CG-S (e) } \\
\mathrm{N}=36\end{array}$ & $\begin{array}{l}\text { P-value } \\
\text { axd }\end{array}$ & axe & & bxc \\
\hline $\begin{array}{l}\text { Age (years) } \\
\text { mean } \pm S D\end{array}$ & $\begin{array}{l}44 \pm 12.1 \\
\mathrm{~N}(\%)\end{array}$ & $\begin{array}{l}43 \pm 13.1 \\
\mathrm{~N}(\%)\end{array}$ & $\begin{array}{l}45 \pm 11.1 \\
\mathrm{~N}(\%)\end{array}$ & $\begin{array}{l}33 \pm 13.1 \\
N(\%)\end{array}$ & $\begin{array}{l}39 \pm 13.8 \\
N(\%)\end{array}$ & $<0.0001^{*}$ & 0.0736 & & 0.6739 \\
\hline female & $11(21)$ & $7(27)$ & $4(15)$ & $39(36)$ & $13(36)$ & & & & \\
\hline male & $42(79)$ & $19(73)$ & $23(85)$ & $70(64)$ & $23(64)$ & & & & \\
\hline $\begin{array}{l}\text { Lifestyle } \\
\text { Smoking } \\
\text { Alcohol }\end{array}$ & $15(28)$ & $7(27)$ & $8(30)$ & $6(6)$ & $1(3)$ & $\begin{array}{l}0.0782 \\
0.0001\end{array}$ & $\begin{array}{l}0.1742 \\
0.0017\end{array}$ & & $\begin{array}{l}0.3265 \\
0.8269\end{array}$ \\
\hline $\begin{array}{l}\text { Consuption } \\
\text { Ilicit drugs }\end{array}$ & $\begin{array}{l}3(6) \\
5(9)\end{array}$ & $\begin{array}{l}2(8) \\
1(4)\end{array}$ & $\begin{array}{l}1(4) \\
4(17)\end{array}$ & $\begin{array}{l}14(13) \\
13(12)\end{array}$ & $\begin{array}{l}0(0) \\
0(0)\end{array}$ & $\begin{array}{l}0.1853 \\
0.9492\end{array}$ & $\begin{array}{l}0.2692 \\
0.0696\end{array}$ & & $\begin{array}{l}0.6104 \\
0.1895\end{array}$ \\
\hline Comorbidities & & & & & & & & & \\
\hline $\begin{array}{l}\text { Hypertension } \\
\text { Diabetes }\end{array}$ & $10(19)$ & $4(16)$ & $6(25)$ & $4(4)$ & $2(6)$ & 0.0014 & 0.0637 & & 0.49262 \\
\hline $\begin{array}{l}\text { Mellitus } \\
\text { Familial History } \\
\text { of }\end{array}$ & $10(19)$ & $5(20)$ & $5(21)$ & $1(1)$ & $1(3)$ & $<0.0001$ & 0.0210 & & 1 \\
\hline Schizophrenia & $32(60)$ & $17(65)$ & $15(56)$ & $15(14)$ & $1(3)$ & $\begin{array}{l}<0.0001 \\
\text { P-value }\end{array}$ & $<0.0001$ & OR $(95 \% \mathrm{CI})$ & 0.5772 \\
\hline $\begin{array}{l}\text { GSTT1 } \\
\text { present }\end{array}$ & $35(66)$ & - & - & 64 (59) & - & & & & \\
\hline null & $18(34)$ & - & - & $45(41)$ & - & & & $\begin{array}{l}1.36 \\
(0.69- \\
2.71)\end{array}$ & \\
\hline $\begin{array}{l}\text { GSTM1 } \\
\text { present }\end{array}$ & $26(49)$ & - & - & $63(58)$ & - & 0.4683 & & & \\
\hline null & $27(51)$ & - & - & $46(42)$ & - & 0.3784 & & $\begin{array}{l}0.70 \\
(0.36- \\
1.36)\end{array}$ & \\
\hline $\begin{array}{l}\text { GSTT1/GSTM1 } \\
\text { present/present }\end{array}$ & $19(36)$ & - & - & $30(28)$ & - & 0.3680 & & $1.47(0.73-2.97)$ & \\
\hline null/null & $11(21)$ & - & - & $12(11)$ & - & 0.1534 & & $2.12(0.87-5.18)$ & \\
\hline present/null & $16(30)$ & - & - & $34(31)$ & - & 0.8967 & & $0.95(0.47-1.95)$ & \\
\hline null/present & $7(13)$ & - & - & $33(30)$ & - & 0.0301 & & $0.35(0.14-0.86)$ & \\
\hline
\end{tabular}

ROC curve analysis showed significant discrimination between patients and controls for MDA levels (TSG - AUC: 0.93; sensitivity: 96\%; specificity: 94\%; cut-off: $146.68 \mathrm{ng} / \mathrm{mL}$; SG-1 - AUC: 0.95; sensitivity: 100\%; specificity: 94\%; cut-off: 146.8 ng/mL; SG-2 - AUC: 0.91; sensitivity: 92\%; specificity: 94\%; cut-off: $146.68 \mathrm{ng} / \mathrm{mL}$; CGOS - AUC: 0.91; sensitivity: 92\%; specificity: $94 \%$; cut-off: $146.68 \mathrm{ng} / \mathrm{mL}$ ), but not for TEAC levels (AUC <0.70).

\section{DISCUSSION}

We found that the combination GSTT1-present/GSTM1-null, whose genes encoding enzymes was associated with an antioxidant effect, and increased MDA plasma levels, a marker of lipid peroxidation, with schizophrenia. Treatment-responsive group and patients with typical and atypical antipsychotic use showed similarity, even in the comparison of oxidative stress markers, possibly due to the ' rigorous patient monitoring and controlled treatment. In this case, it should be expected worse conditions mainly in the treatment- 
resistant group considering the type and period of treatment with atypical antipsychotic. Other researchers have pointed out that this combination can alter antioxidant enzyme levels, increasing lipid peroxidation and consequently oxidative damage in the brain (Pinheiro et al., 2017).

The prevalence of familial history of schizophrenia adds relevance to genetic factors for the disease (Pinheiro et al., 2017). In this context, the association of the GSTMI genotype in East Asian patients with schizophrenia, but not for GSTT1 (Kim et al., 2015) is emphasized, whereas in our study the genotypes of the two polymorphisms were similar between the groups. On the other hand, in this series a lower frequency of combination GSTT1-null/GSTM1-present was found in patients compared to controls, in disagreement with two meta-analyses (Kim et al., 2015; Koga et al., 2016). It is possible that the presence of one of these genotypes could attenuate the impairment caused by the absence of the other, explaining the differences in clinical profile and response to drug treatment (Pinheiro et al., 2017), which should be investigated in further studies on gene-gene and geneenvironment interactions (Hu et al., 2016).

In addition, the association of oxidative stress and psychiatric disorders is also highlighted (Boskovic et al., 2011). Regarding oxidative stress biomarkers, plasma MDA levels were higher in patients with schizophrenia than in controls, as reported in other studies (Liencres et al., 2014; Reyezuddin et al., 2014). In this case, increased MDA levels were observed mainly in patients with the combination GSTT1-present/GSTM1-null genotypes. It is emphasized that MDA, end-product of lipid peroxidation in phospholipids membrane, is released into extracellular matrix and reaches the blood flow (Liencres et al., 2014; Reyezuddin et al., 2014). In this way, high generation of reactive oxygen species and, consequently, higher MDA level can cause neuronal damage (Liencres et al., 2014), and could be aggravated by genetic variants of GSTs that may impair its antioxidant effect.

Furthermore, in this series MDA levels showed high sensibility and specificity, indicating discriminatory power and a potential prognostic tool for oxidative stress in patients with schizophrenia, according to another study (Pawar and Abhang, 2017). In contrast, similar values of TEAC, which correspond to the antioxidant capacity and protection against free radicals and reactive oxygen species (Gilca et al., 2014; Filho et al., 2018), were showed in all groups, but reduced level in patients using-antipsychotics was also reported (Gilca et al., 2014).

In addition, smoking, as well as SAH and DM also in another studies (Gilca et al., 2014; Sun et al., 2018), prevailed in the patients compared to controls. There an increasing cognitive effect of smoking, which may be responsible for minimizing the symptoms of the disorder and increasing the metabolism of antipsychotic drugs (Gage and Munafò, 2015). On the other hand, toxic chemicals and oxidative compounds, such as free radicals and nitric oxide, in gas phase are present in high concentrations in tobacco, which contributes to increase oxidative stress (Pawar and Abhang, 2017), as well as the use of atypical antipsychotics (Gilca et al., 2014; Sun et al., 2018). In relation to DM and SAH, these may be related to weight gain induced by antipsychotic agents and to altered glucose tolerance rates (Sun et al., 2018). These comorbidities may cause a metabolic syndrome, ranging from $11 \%$ to $69 \%$ in patients with schizophrenia; estimated at $32.5 \%$ in a meta-analysis study (Freitas et al., 2016).

This study was based on a transversal analysis of a unique peripheral blood sample of a reduced number of patients, which should be considered a limitation, although in other 
studies the same methodology was also used even with reduced series (Gilca et al., 2014; Liencres et al., 2014). A longitudinal analysis with serial samples of peripheral blood could increase reliability. Furthermore, the oxidative stress biomarker levels could vary according to several pathophysiological processes, but the presence of a control group as we presented can contribute to data analyses. In addition, the specific impact of antipsychotic drugs on the level of MDA and TEAC should also be developed in further studies. Also, it is necessary to clarify whether MDA and TEAC plasma concentration are driven by the schizophrenia or if these markers have some influence on the development of this disorder.

\section{CONCLUSIONS}

The lower frequency of the combination GSTT1-null/GSTM1-present genotypes in patients suggests exposure to oxidative stress and increased level of MDA, mainly aggravated by smoking, SAH, DM and familial history of schizophrenia. Furthermore, high sensitivity and specificity of plasma MDA indicates its potential as a marker of oxidative stress in schizophrenia.

\section{ACKNOWLEDGMENTS}

We thank the CNPq (National Council for Scientific and Technological Development) for financial support as well as FAMERP (Medical School of São José do Rio Preto, SP), NPBIM (Research Center in Biochemistry and Molecular Biology FAMERP) and LQBOA (Bio-Organic and Environmental Chemistry LaboratoryIBILCE/UNESP) for supporting the research.

\section{CONFLICTS OF INTEREST}

The authors declare no conflict of interest.

\section{REFERENCES}

Bošković M, Vovk T, Kores Plesničar B and Grabnar I (2011). Oxidative stress in schizophrenia. Curr. Neuropharmacol. 9: 301-312. doi:10.2174/157015911795596595

Emiliani FE, Sedlak TW and Sawa A (2014). Oxidative stress and schizophrenia: recent breakthroughs from an old story. Curr. Opin. Psychiatry. 27: 185-190. doi:10.1097/YCO.0000000000000054

Filho GMA, Martins DP, Lopes AM, Brait BJ, et al. (2018). Oxidative stress in patients with refractory temporal lobe epilepsy and mesial temporal sclerosis: Possible association with major depressive disorder. Epilepsy Behav. 80: 191-196. https://doi.org/10.1016/j.yebeh.2017.12.025

Freitas PHB, Nunes FDD, Pinto JAF, Sousa PHA, et al. (2016). Metabolic Syndrome in Patients with Refractory Schizophrenia: Sociodemographic and Clinical Characteristics. R. Enferm. Cent. O. Min. 1: 1976-93. http://dx.doi.org/10.1590/1982-0194201600009

Gage SH and Munafò MR (2015). Rethinking the association between smoking and schizophrenia. Lancet Psychiatry. 2: 118-119. https://doi.org/10.1111/jan.12527

Gilca M, Piriu G, Gaman L, Delia C, et al. (2014). A study of antioxidant activity in patients with schizophrenia taking atypical antipsychotics. Psychopharmacology. 231: 4703-4710. https://doi.org/10.1007/s00213-014-3624-0

Hu B, Huang H, Wei Q, Ren M, et al. (2019). Transcription factors CncC/Maf and AhR/ARNT coordinately regulate the expression of multiple GSTs conferring resistance to chlorpyrifos and cypermethrin in Spodoptera exigua. Pest. Manag. Sci. 75: e5316.https://doi.org/10.1002/ps.5316

$\mathrm{Hu}$ W, Chen M, Wu W, Lu J, et al. (2016). Gene-gene and gene-environment interactions on risk of male infertility: Focus on the metabolites. Environ. Int. 91: 188-195. http://dx.doi.org/10.1016/j.envint.2016.02.025 
Kane J, Honigfeld G, Singer J and Meltzer H (1988). Clozapine for the treatment-resistant schizophrenic: a double-blind comparison with chlorpromazine. Arch. Gen. Psychiatry. 45(9): 789-796.

Kim SK, Kang SW, Chung JH, Parque HJ, et al. (2015). Genetic Polymorphisms of Glutathione-Related Enzymes (GSTM1, GSTT1, and GSTP1) and Schizophrenia Risk: A Meta-Analysis. Int. J. Mol. Sci. 16: 19602-19611. https://doi.org/10.3390/ijms160819602

Koga M, Serritella AV, Sawa A and Sedlak TW (2016). Implications for reactive oxygen species in schizophrenia pathogenesis. Schizophr. Res. 176: 52-71. http://dx.doi.org/10.1016/j.schres.2015.06.022

Liencres CG, Tas C, Brown EC, Erdin S, et al. (2014). Oxidative stress in schizophrenia: a case-control study on the effects on social cognition and neurocognition. BMC Psychiatry. 268(14). https://doi.org/10.1186/s12888-014$0268-\mathrm{x}$

Mak M, Samochowiec J, Frydecka D, Pełka-Wysiecka J, et al. (2019). First-episode schizophrenia is associated with a reduction of HERV-K methylation in peripheral blood. Psychiatry Res. 271: 459-463. https://doi.org/10.1016/j.psychres.2018.12.012

Nakamura T, Ohnuma T, Hanzawa R, Takebayashi Y, et al. (2015). Associations of common copy number variants in glutathione S-transferase mu 1 and D-dopachrome tautomerase-like protein genes with risk of schizophrenia in a Japanese population. Am. J. Med. Genet. B. Neuropsychiatr. Genet. 168: 630636.https://doi.org/10.1002/ajmg.b.32347

Pawar MRS and Abhang AS (2017). Consumption of GSH with the Increase in Oxidative Stress in Chronic Obstructive Pulmonary Disease (COPD) Patients. Int. J. Cur. Res. Rev. 9: 25-29.

Pellegrini N, Proteggente A, Pannala A, Yang M, et al. (1999). Antioxidant activity applying an improved ABTS radical cation decolorization assay. Free Radic. Biol. Med. 26: 1231-1237.

Pinheiro DS, Santos RS, Brito RB, Cruz AHS, et al. (2017). GSTM1/GSTT1 double-null genotype increases risk of treatment-resistant schizophrenia: A genetic association study in Brazilian patients. Plos One. 12: e0183812. https://doi.org/10.1371/journal.pone.0183812

Reyazuddin M, Azmi AS, Islam N and Rizvi A (2014). Oxidative stress and level of antioxidant enzymes in drug-naïve schizophrenics. Indian J. Psy. 56: 344-349.

Rossini A, Rapozo DCM, Amorim LMF, Macedo JMB, et al. (2002). Frequencies of GSTM1, GSTT1 and GSTP1 polymorphisms in a Brazilian population. Genet. Mol. Rev. 1: 233- 240.

Salazar LA, Hirata MH, Cavalli SA, Machado MO, et al. (1998). Optimized Procedure for DNA isolation from fresh and cryopreserved clotted human blood useful in clinical molecular testing. Clin. Chem. 44: 1748-1750.

Shojaeimotlagh V, Hashiehbaf A, Karami M, Monjazebi F, et al. (2019). Prevalence of metabolic syndrome in Iranian patients with schizophrenia: A systematic review and meta-analysis. Diabetes Metab. Syndr. 13: 143-147. https://doi.org/10.1016/j.dsx.2018.08.014

Sun L, Getz M, Daboul S, Jay M, et al. (2018). Independence of diabetes and obesity in adults with serious mental illness: Findings from a large urban public hospital. J. Psychiatr. Res. 99: 159-166. https://doi.org/10.1016/j.jpsychires.2018.01.005

Zheng W, Zhu W, Feng Z, Liang Q, et al. (2019). Increased serum malondialdehyde levels are associated with grey matter volume loss in patients with non-alcoholic cirrhosis. Quant. Imaging Med. Surg. 9: 230-237. http://dx.doi.org/10.21037/qims.2018.12.12 